\title{
Le Systéme D'encadrement Pédagogique des Futurs Enseignants D'eps: Une Lecture de la Gestion des Rétroactions des Inspections Pédagogiques à L’injeps/Bénin
}

\author{
Attiklemé Olivier, \\ Agbodjogbe Basile, \\ Atoun Carlos, \\ Attiklemé Kossivi,
} Laboratoire des didactiques des Disciplines (LDD), Université d'Abomey-Calavi, République du Bénin

\section{Pr. Kpazaï Georges,}

Groupe de Recherches sur l'Évaluation et le Développement des Compétences en Activité Physique et en Santé (GRÉDCAPS), École des sciences de l'activité physique, Université Laurentienne, Sudbury (ON), Canada

\section{Résumé}

Le but de cette recherche est d'analyser le système d'encadrement pédagogique des étudiants en Sciences et Techniques des Activités Physiques et Sportives (STAPS) en formation initiale à l'Institut National de la Jeunesse, de l'Éducation Physique et des Sports (INJEPS) lors de leurs stages dans des établissements d'enseignement secondaire. À partir d'une grille de lecture composée du modèle systémique du processus d'intervention de Dunkin et Biddle (1974) et celui de Chevallard (1992), cette étude problématise d'une part, le fait didactique à partir des variables de contexte, de programme, de processus et de produit, et d'autre part du concept de praxéologie. Par ailleurs, elle cherche aussi à comprendre les variables déterminantes au processus de supervision pédagogique et la manière dont l'INJEPS gère les feedbacks produits par les superviseurs pédagogiques de l'INJEPS et de la Direction de l'Inspection Pédagogique. Il ressort, de l'analyse des résultats des investigations, que les superviseurs pédagogiques des deux institutions mettent essentiellement l'accent sur les variables de contexte, de processus et de produit lors de l'encadrement pédagogique des stagiaires de 3ème , 4ème et 5ème année, alors que les variables de programme aussi sont prises en compte en 1ère et 2ème année. A l'issue des supervisions, seules les notes de 
stage produites par les rapports pédagogiques des superviseurs sont prises en compte par l'administration lors des évaluations partielles et terminales des étudiants. Aucun traitement didactique des contenus des feedbacks n'est fait dans la perspective d'améliorer la formation pédagogique des futurs enseignants.

Mots clés: Supervision pédagogique, Stage, Feedback, Éducation Physique et Sportive

\title{
The Management of the Pedagogical System of the Preservice Physical Education Teachers : A Reading of Educational Inspectors` Feedbacks in INJEPS/ Repubic of Benin
}

\author{
Attiklemé Olivier, \\ Agbodjogbe Basile, \\ Atoun Carlos, \\ Attiklemé Kossivi,
}

Laboratoire des didactiques des Disciplines (LDD),

Université d'Abomey-Calavi, République du Bénin

Pr. Kpazaï Georges,

Groupe de Recherches sur l'Évaluation et le Développement des

Compétences en Activité Physique et en Santé (GRÉDCAPS),

École des sciences de l'activité physique, Université Laurentienne,

Sudbury (ON), Canada

\begin{abstract}
The aim of this research is to analyze the pedagogical management system of students in Science and echnology of Physical and Sports Activities in initial training at the National Institute of Youth, Physical Education and Sports (INJEPS) during their internships in secondary schools. From a reading grid composed of the systemic model of the intervention process of Dunkin and Biddle (1974) and that of Chevallard (1992), this study problematizes on the one hand the didactic fact based on the variables of context, program, process and product and on the other hand the concept of praxeology. It also seeks to understand the key variables in the pedagogical supervision process and how the INJEPS manages the feedback produced by the supervisors of the
\end{abstract}


INJEPS and the Pedagogical Inspectorate. From the analysis of the results of the investigations, it emerges that the supervisors of the two institutions focus mainly on the context, process and product variables during the pedagogical supervision of the trainees of the 3rd, 4th and 5th year, while the program variables are also taken into account in the first and second year. At the end of the supervisions, only the internship notes produced by the supervisors' teaching reports are taken into account by the administration during the partial and final evaluations of the students. No didactic treatment of the content of the feedbacks is done with a view to improving the pedagogical training of future teachers.

Keywords: Pedagogical supervision, Internship, Feedback, Sport and Physical Education

\section{Introduction}

La question de la formation continue des enseignants est sans nul doute l'un des paradoxes les plus remarquables du moment où les continents semblent placer leur espoir dans l'édification d'une «société de connaissance »(Baillat, Niclot, \& Ulman, 2010). Force est aussi de constater la modestie des efforts que certains États comme le Bénin consentent en faveur de la formation.

Ce paradoxe renvoie à la complexité d'une question qui convoque de multiples dimensions qui sont, entre autres, disciplinaires, didactiques, pédagogiques et professionnelles. Cette complexité suscite des tensions fortes entre d'une part la logique de l'éducation et de la formation, et d'autre part entre celle de la formation générale et professionnelle où la gestion de la visée « suivie-évaluation » reste problématique.

Dans cette perspective, s'inscrit l'élaboration des référentiels des offres de formation, le référentiel «suivie-évaluation » où le système de relation mis en place prend en compte les compétences de l'enseignant. Celuici doit avoir les qualités nécessaires pour favoriser la transmission des savoirs qu'il détient. Il contraint les autorités politico-administratives à mettre en place un système de supervision pédagogique visant à suivre et à aider les futurs enseignants dans l'accomplissement de leur tâche. Ce système est relatif à toutes les disciplines y compris l'Éducation Physique et Sportive. En matière de supervision pédagogique de l'enseignement, les recherches montrent que les enseignants ont des préférences diverses par rapport au type d'aide à recevoir en pédagogie (Brunelle, Drouin, Godbout \&Tousignant, 1988 ; Glattorn, 1984).

En effet, la supervision pédagogique vise le respect des méthodes d'enseignement et des critères rigoureux et spécifiques à observer par l'enseignant en situation de classe. Les acteurs de ce système sont issus de 
diverses structures. Au Bénin, ils émanent prioritairement de l'Inspection Générale Pédagogique du Ministère (IGPM) et de l'Institut National de la Jeunesse, de l'Éducation Physique et du Sport (INJEPS). Plusieurs idées sont faites au sujet de la supervision pédagogique.

Pour certaines personnes, la supervision pédagogique est perçue comme une séance d'observation de classe et de critiques où le superviseur s'acharne sur l'enseignant, ce qu'il a fait de mal, son comportement ou son attitude en situation de classe. D'autres par contre, admettent que c'est un moment privilégié pour le superviseur pédagogique d'étaler ses connaissances sans tenir compte de la prestation de l'enseignant. S'inscrivant dans une approche descendante de respect des normes curriculaires, certains enseignants considèrent la supervision pédagogique comme contraignante et d'autres la rejettent systématiquement. Il y en a même qui trouvent que c'est un moment propice pour échanger avec le superviseur en vue de s'approprier de nouvelles méthodes, de nouvelles compétences ; c'est aussi le moment pour eux de se corriger en vue d'améliorer leurs prestations (Issak, 2005).

C'est ainsi que dans le cadre de l'amélioration de la qualité de l'enseignement, plusieurs séries d'inspection sont faites à l'endroit des enseignants notamment en EPS selon leur statut (Agents permanents de l'État, vacataires, Stagiaires).

En EPS, au Bénin, à l'image d'un bon nombre de pays, les Activités Physiques et Sportives (APS) constituent les moyens qu'utilise l'enseignant d'EPS pour mener à bien son action éducative auprès des élèves ; d'où la nécessité de maîtriser un savoir-faire et un savoir relatifs aux méthodes d'enseignement dans cette discipline. Le savoir-faire doit être réactualisé avec l'évolution des sciences, en vue de développer les stratégies nécessaires d'intervention. Dans le cadre du suivi pédagogique des étudiants de l'INJEPS, les autorités académiques, afin de veiller au respect de l'éthique enseignante, font appel aux superviseurs pédagogiques, notamment à ceux de l'INJEPS et à ceux de l'IGPM (Inspection Pédagogique Générale du Ministère), pour assurer l'encadrement pédagogique des étudiants stagiaires placés en position d'enseignant dans les différents collèges et lycées.

Comment se déroule alors cet encadrement pédagogique ? Quelles sont les critères utilisés par ces superviseurs pédagogiques pour évaluer ces stagiaires ? Ces critères sont-ils objectifs ? Que font-ils des feedbacks à la fin des inspections ou des visites pédagogiques en vue d'améliorer la pratique enseignante des futurs enseignants ? Ce sont ces questions que traite cet article dont l'objectif est d'analyser, dans un premier temps, le système d'encadrement pédagogique des stagiaires de l'INJEPS et principalement les feedbacks fournis à ces derniers à la suite des visites de classes ou d'inspections pédagogiques ; dans un deuxième temps, il s'agit d'analyser aussi le contenu des savoirs véhiculés lors des rétroactions des superviseurs 
pédagogiques dans le contexte de la formation pratique des étudiants. Autrement dit, il s'agit d'une part, d'analyser au plan pédagogique le système d'évaluation de la pratique enseignante des étudiants-stagiaires afin de comprendre sa pertinence et d'autre part, au plan administratif de comprendre comment ces feedbacks sont utilisés en vue d'améliorer la qualité de l'enseignement. Dans cette perspective, trois sections structurent cet article. Après avoir présenté le contexte institutionnel de l'organisation des inspections pédagogiques à l'INJEPS, il sera présenté les attributions des acteurs des deux institutions ayant en charge les visites d'inspection. Dans une deuxième section nous introduirons la problématique essentielle de l'étude en s'appuyant sur un double ancrage théorique : la conception systémique du processus d'intervention de Dunkin et Biddle (1974) adaptée par Brunelle et al. (1988) et la théorie anthropologique du didactique de Chevallard (1989). Une troisième partie discutera les résultats et soulignera les issues des feedback reçus par les étudiants-stagiaires au regard de la nature des savoirs véhiculés par ces derniers.

\section{Contexte institutionnel de la formation et de la gestion pédagogique des étudiants à l'INJEPS}

Comme nous l'avions montré dans la note introductive, rappelons-le deux institutions interviennent dans les inspections pédagogiques des étudiants en formation initiale à l'INJEPS : l'INJEPS et l'IGPM. Ces deux institutions sont dotées d'organes de contrôle et de gestion des visites de classe qu'il s'avère indispensable de décrire.

\subsection{Les organes de l'INJEPS}

Selon l'article 30 du décret $N^{\circ}$ 96-550 du 6 décembre 1996, six organes assurent la gestion de la formation à l'INJEPS au rang desquels se figurent le conseil pédagogique et le conseil des enseignants du département. L'une des principales fonctions du conseil pédagogique est la conception, la sanction des études, ainsi que la définition des modalités de contrôle. Il est renforcé dans ses attributions par le conseil des enseignants du département qui, sur les instructions $\mathrm{du}$ président du conseil pédagogique organise les stages pédagogiques (Cf. article 5 du décret ci-dessus référencé).

\subsubsection{L'organisation des formations à l'INJEPS}

Deux mentions accueillent les étudiants à l'INJEPS dans le domaine des sciences de l'éducation et de formation en l'occurrence les STAPS qui constituent la préoccupation de notre étude. Elles disposent de cinq spécialités : 1' enseignement de l'EPS ou APSA et motricité (qui prépare à la profession d'enseignement d'EPS) ; l'entrainement sportif (qui prépare à la profession d'éducateur sportif notamment - d'entrainement de haut niveau); 
le Handisport (qui prépare au métier d'éducateur sportif auprès des personnes vivant des handicaps) ; le Sport pour tous (qui prépare à l'encadrement du sport de masse) ; le Management du sport (qui prépare à l'organisation, à la planification, à la gestion du marketing du sport). La spécialité qui nous concerne dans le cadre de cet article est celle qui prépare à la profession d'enseignement d'EPS.

\subsubsection{L'organisation des stages}

L'INJEPS est au cœur de la formation des futurs enseignants et entraîneurs. Pour assurer le suivi pédagogique de ses étudiants, la direction académique prévoit des stages d'enseignement de l'EPS dans les établissements primaires (pour les étudiants de 1ère année) et secondaires (pour les étudiants de 2ème, 3ème 4ème et 5ème année ayant l'option « enseignement »). Seuls les étudiants qui ont l'option « entraînement » font leur stage dans les clubs et associations sportives (AS) à partir de la 3ème année. En option « enseignement», cette pratique pédagogique se déroule sur six mois (de novembre à avril) chaque année, en continue ou en alternance (selon les promotions) c'est-à-dire, un semestre avec la formation académique qui dure trois ou cinq ans selon que l'on postule à la licence ou au Master. Elle s'effectue aussi sur un semestre pour les étudiants en 5ème année ou en année de Certificat d'Aptitude au Professorat d'EPS (CAPEPS) qui équivaut au Master (S3, S4) professionnel, à raison de 6 mois de cours, avec $2 \mathrm{~h}$ d'Animation Pédagogique (AP) et $2 \mathrm{~h}$ d'Association Sportive (AS). Pour les autres stagiaires, elle est couplée hebdomadairement avec la formation académique et a lieu chaque année : a) les mardis de $7 \mathrm{~h}$ à $10 \mathrm{~h}$ et de $16 \mathrm{~h}$ à $19 \mathrm{~h}$ pour les stagiaires qui sont en 2ème année de formation (les étudiants de 2 ème année de Licence), conformément au système Licence Master Doctorat (LMD) ; b) les jeudis de $7 \mathrm{~h}$ à $10 \mathrm{~h}$ et $16 \mathrm{~h}$ à $19 \mathrm{~h}$ pour les stagiaires qui sont en 3ème année de formation (les étudiants en année de Licence, conformément au système LMD) ; c) les stagiaires de la 4ème année de formation (1ère année de Master) vont remplacer au second semestre ceux de la 5ème année (2ème année de Master), c'est-à-dire à partir du mois de Mars ; d) les stagiaires de la 5ème année (Master, S3, S4) font les cours dans les collèges du mois d'Octobre au mois de Février. Des superviseurs pédagogiques nommés par l'INJEPS dans ce cadre de formation, sont chargés de la supervision pédagogique des stagiaires, c'est-à-dire du suivi pédagogique et du contrôle de leur stage.

\subsection{Les organes de L'IGPM}

Le système éducatif béninois est caractérisé actuellement par trois ordres d'enseignement : le MEMP, le MESFTP et le MESRS. Dans le cadre de notre étude, c'est le MESFTP (Ministère de l'Enseignement Secondaire, de 
la Formation Technique et Professionnelle) qui nous intéresse. Il est l'organe qui définit les grands projets de l'enseignement secondaire et dispose en son sein d'une Direction d'Inspection Pédagogique encore appelée « L'Inspection Générale Pédagogique du Ministère (IGPM)». Cette direction a une structuration bien hiérarchisée, une mission claire et des moyens, ce qui lui permet de mieux accomplir ses tâches.

\subsubsection{L'Inspection Générale Pédagogique du Ministère (IGPM)}

C'est un organe d'inspection et d'innovation pédagogique, de contrôle dans le sous-secteur de l'Enseignement Secondaire Général et de la Formation Technique et Professionnelle créé par le Décret n²012-431 du 06 Novembre 2012. L'arrêté n’337/MESFTPRIJ/DC/SGM/IGPM/SA du 23 juillet 2013 en détermine les attributions, l'organisation et le fonctionnement. Au nombre de ses multitudes d'actions (veiller à la qualité de l'enseignement et contrôle la gestion pédagogique des établissements publics et privés d'enseignement secondaire général et de formation technique et professionnelle, contrôle et gestion des évaluations, etc.) qui relèvent de ses attributions ; celles qui nous concernent le plus sont les actions de contrôles et d'évaluation des enseignants.

\subsubsection{Les actions de contrôles et d'évaluation de l'IGPM}

Les actions de contrôle concernent essentiellement le contrôle de : a) l'organisation de l'enseignement au plan quantitatif et qualitatif (les plannings, les cahiers de textes ou les comptes rendus d'activités sont des indicateurs à consulter.) ; b) l'utilisation des crédits et matériels alloués à l'établissement ; c) l'état des matériels et installations utilisés pour l'enseignement.

Quant aux actions d'évaluation, elles concernent l'évaluation de la valeur professionnelle des agents d'exécution du ministère (professeurs, conseillers). Pour cela, il lui faudra apprécier le travail effectif au plan quantitatif et qualitatif non seulement lors de sa visite mais pour toute la période antérieure, des capacités d'évolution de l'enseignant et donc juger si le poste occupé convient à ses aptitudes.

C'est donc au vu de ces attributions que l'INJEPS fait recours à ces inspecteurs et conseillers pédagogiques de l'IGPM, pour une assistance pédagogique dans la supervision de ses étudiants. Dans cette perspective quels sont les moyens dont disposent ces acteurs pour accomplir leur mission?

\subsection{Les outils de collecte des informations}

Pour les deux catégories d'acteurs plusieurs moyens sont mis à leur disposition pour bien assurer ce suivi : les fiches ou grilles d'inspection comportant des critères et indicateurs d'appréciation. Les critères utilisés abordent diverses catégories d'aspects à savoir : 
- la tenue des documents pédagogiques, l'organisation spatiale et la gestion temporelle de la classe, la démarche méthodologique, etc. ;

- les entretiens pré et post séance. Les entretiens post sont communément appelés des feedbacks. Cependant il faut noter qu'il existe une légère disparité au niveau des grilles utilisées par les acteurs des deux institutions. Cette disparité est due aux cibles d'intervention de ces deux institutions : l'une se chargeant de la formation initiale (INJEPS) et l'autre de la formation continue (IGPM). L'autre aspect de la disparité qui existe réside aussi au niveau de la qualification professionnelle des acteurs de la supervision pédagogique. Pour l'IGPM, ce sont des inspecteurs et parfois des conseillers pédagogiques. Quant aux acteurs de l'INJEPS, ils sont pour la plupart des enseignants-chercheurs ayant un profil pédagogique avéré. Cette différenciation des superviseurs pourrait, toutefois, influencer les résultats de la supervision pédagogique des étudiants. Il se pose ainsi la question de la pertinence des critères utilisés mais aussi de la nature des feedbacks réalisés.

\section{Cadre théorique, problématique et questions de recherche}

Plusieurs études ont abordé la supervision et la gestion des feedbacks. Maintes fonctions sont dévolues à la supervision. Un consensus général se dégage des travaux scientifiques sur ce thème sur le fait que la supervision permet d'améliorer la qualité de l'enseignement, de maîtriser des connaissances et d'acquérir des habiletés et conséquemment d'améliorer la professionnalité enseignante (Brunelle et al, 1988 ; Glatthorn, 1984, Boulet et Rousseau, 2002 ; Olivia et Pawlas, 2004 ; Nolan et Hoover, 2011.). Pour certains savants du domaine de la supervision, dans la quête d'une clarification de ce concept, il semble exister des connotations contradictoires qui expriment à la fois l'idée de surveillance, de contrôle, de régulation et aussi d'accompagnement, voire de l'instruction d'un certain leadership (SnowGerono, 2008; Villeneuve, 1994). La dimension dialogique du concept est mise en exergue par Brunelle et al. (1988) lorsqu'ils pointent des problèmes que peuvent générer l'issue de la supervision lors de l'analyse du vécu pédagogique qui met en communication deux personnes devant s'inscrire à priori dans une synergie d'actions.

En EPS, la supervision pédagogique renvoie à deux ordres de rapports : 1) les rapports qui lient les structures organisationnelles et infrastructurelles de la supervision (Ministère de l'enseignement secondaire) aux structures dans lesquelles celle-ci s'exécute, c'est-à-dire les structures d'accueil (les établissements) et 2) les relations entre les superviseurs pédagogiques et les enseignants d'EPS avec les responsables académiques de cette structure d'accueil à savoir les enseignants des disciplines autres que l'EPS.

En s'inscrivant dans cette même logique, Boutet et Rousseau (2002) établissent trois «cycles de supervisions »: 1) le premier consiste pour le 
superviseur à identifier les difficultés de l'enseignant, 2) le second est consacré à une période d'observation en classe pendant laquelle le superviseur doit tenir compte du problème posé par l'enseignant et 3) le troisième a lieu lors de rencontre post-classe. Dans cette partie, le superviseur consacrera le temps à l'enseignant supervisé pour confronter ce qui a été observé en classe à ce qui a été planifié. Selon ces auteurs, c'est ce temps de confrontation qui rend possible les rétroactions.

Cette troisième étape très importante rend compte beaucoup plus de la gestion des rétroactions voire des feedbacks issus de l'observation par les protagonistes de l'action didactique. Elle interpelle les acteurs de la supervision des étudiants en formation initiale à l'INJEPS en termes de stratégie de supervision pour constater Comment ces derniers gèrent les différentes variables d'interventions observées et par ricochet les différents savoirs issus des feedbacks générés par les pratiques enseignantes des étudiants. C'est dans cette perspective que cet article, sous le couvert d'un double ancrage théorique à savoir, le modèle d'intervention de Dunkin et Biddle (1974) repris par Brunelle et al. (1988) qui explique les différentes variables permettant d'analyser les contenus des feedbacks en activité physique et la théorie anthropologique du didactique de Chevallard (1992) qui cherche à comprendre et à expliquer comment se font les supervisions des étudiants en formation initiale à l'INJEPS, est réalisé. Autrement dit, il s'agit sous couvert de ce modèle composite, d'approcher à travers l'analyse des feedbacks produits, à la suite des inspections, les savoirs véhiculés par ces feedbacks (savoirs professionnels et institutionnels), les types d'objectifs ou de compétences, les directives pédagogiques, les modes d'organisation, d'enseignement et d'évaluation(variables programme et contexte) mais surtout les critères d'évaluation utilisés et la gestion de ces feedbacks du point de vue administratif, pédagogique, voire institutionnel, en terme de praxéologie.

En considérant, à l'instar d'Attiklemé et Kpazaï (2010), que la supervision pédagogique est une action didactique continue d'information, d'instruction, d'orientation, de guidance et de correction des erreurs qu'exerce le superviseur pédagogique sur l'enseignant afin de valoriser davantage sa performance pédagogique, cette étude s'interroge sur la nature des contenus des feedbacks produits par les superviseurs pédagogiques desdites institutions pour comprendre leurs utilités aux plans pédagogique, administratif et institutionnel (Brunelle et al., 1988).

Il s'agit, en référence à ce modèle d'appréhender la praxéologie didactique des savoirs mis en jeu lors des feedbacks, d'analyser les contenus de ces feedbacks produits par les superviseurs pédagogiques des deux institutions. Au vue de cette problématique, cette étude aborde les questions de recherche ci-après : 
1) Quelle est la nature des savoirs des feedbacks produits par chaque catégorie de superviseurs pédagogiques?

2) Quels sont les savoirs mobilisés pour améliorer les prestations pédagogiques des enseignants d'EPS ?

3) Quelles sont les activités de remédiation envisagées par ces feedbacks en terme de formation pratique des enseignants d'EPS.

\section{Cadre méthodologique}

Les catégories d'acteurs concernées par cette étude sont des enseignants-chercheurs de l'INJEPS, les inspecteurs et conseillers pédagogiques de l'IGPM, et les étudiants en formation initiale à l'INJEPS. Ils constituent alors la population d'enquête.

\section{1. Échantillonnage}

Pour retenir les sujets de notre échantillonnage, nous avons fait un choix raisonné. Il s'agit d'un échantillonnage fondé sur l'étude de la compréhension des gestions des supervisions pédagogiques et de la nature des contenus des feedbacks réalisés. Cet échantillonnage nous a permis de retenir cinq différentes catégories d'acteurs.

\subsubsection{Les enseignant-chercheurs de l'INJEPS}

Les enseignants-chercheurs de l'INJEPS sont au nombre de dix dont trois sont chargés directement de la gestion des fiches d'inspection pédagogique des étudiants.

\subsubsection{Les inspecteurs pédagogiques de l'EPS}

Selon leur répartition sur le plan national, chaque inspecteur a à sa charge le suivi pédagogique des enseignants et stagiaires d'un département. Ainsi, ce sont les inspecteurs des quatre départements qui sont concernés

\subsubsection{Les conseillers pédagogiques}

Huit conseillers pédagogiques sont choisis à raison de deux par départements pour prendre part à cette étude. Il s'agit des conseillers pédagogiques des départements du Plateau, de l'Ouémé, du Littoral et de l'Atlantique considérés comme les départements phares du Bénin parce qu'ils ont une tradition en matière de gestion des Conseillers Pédagogiques. Ces conseillers ont a priori un rapport favorable aux visites de classe.

\subsubsection{Les étudiants de l'INJEPS}

Ils sont tous du secteur STAPS et sont issus des cinq différentes promotions de l'INJEPS. Nous avons choisi les vingt meilleurs en fonction des notes obtenues en pédagogie pratique dans chaque promotion. Soit au 
total, cent (100) étudiants issus des filières EPS (Éducation Physique et Sportive) et ES (Entraînement sportif). Il s'agit de sujets d'étude ayant un engouement pour la pédagogie pratique et dont les débats interactifs engagés (les feedbacks) sont bien retranscrits dans leurs cahiers bilan. Le tableau cidessous présente la taille de l'échantillon (nombre total des sujets d'étude).

Tableau 1 : Récapitulatif de l'échantillon

\begin{tabular}{|l|l|}
\hline Statut & Nombre \\
\hline Enseignants de l'INJEPS & 03 \\
\hline Inspecteurs & 04 \\
\hline Conseillers pédagogiques & 08 \\
\hline Etudiants de l'INJEPS & 100 \\
\hline Totaux & 115 \\
\hline
\end{tabular}

\subsection{La collecte des données}

Elle est faite grâce à une autorisation de recherche de l'administration de l'INJEPS. Elle a permis de faire une enquête par questionnaire destinée aux étudiants.

Nous avons eu aussi l'accord de principe de ces professeurs certifiés d'EPS en situation de classe qui ont accepté de répondre au questionnaire et nous accorder également un temps pour l'entretien. Nous avons contacté les conseillers pédagogiques, les inspecteurs pédagogiques de l'EPS et les enseignants de l'INJEPS pour avoir leur accord pour le protocole de collecte des données. Tous ont accepté se rendre disponibles pour répondre à nos préoccupations. Enfin, une dernière étape de ce recueil de données a consisté à expliquer aux différents participants, l'absence de tout jugement de valeur de leurs réponses et surtout le respect de l'anonymat des différents résultats.

\subsubsection{Les techniques et outils de collecte de données}

Deux techniques ont permis de collecter les données : l'enquête et l'entretien individuel ;

\subsubsection{L'enquête}

Elle a été réalisée à l'aide d'un questionnaire au niveau des étudiants en formation initiale. Ce questionnaire est adressé aux étudiants et a permis d'avoir des informations relatives au dispositif de formation, d'organisation de déroulement des stages pédagogiques et de la gestion des feedbacks. L'étape de la distribution du questionnaire a été aussi une occasion d'une part, pour recueillir les cahiers de bilans des étudiants (cahiers dans lesquels les rapports des feedbacks sont transcrits) et d'autre part d'avoir des informations complémentaires sur ces feedbacks à savoir : les acteurs (superviseurs pédagogiques) et leurs structures d'appartenance, les contenus des feedbacks non-inscrits dans les cahiers de bilan et les recommandations des superviseurs pédagogiques. 


\subsubsection{L'entretien}

L'entretien est réalisé à l'aide d'un guide d'entretien. Il a été enregistré à l'aide d'un dictaphone. Il est semi-directif et est accordé particulièrement aux Conseillers pédagogiques investis (professeurs certifiés d'EPS), aux inspecteurs et aux enseignants chercheurs de l'INJEPS. Le but visé est de recueillir chez ces participants ce qui fait l'objet de leur feedback et surtout sa nature. Il s'agit aussi d'un entretien visant à recueillir leur avis sur la manière dont ces feedbacks sont conduits, leurs finalités pédagogique et administrative.

\subsection{Le traitement des données}

Le traitement des données recueillies est réalisé en deux étapes : 1) la première étape a permis de retranscrire l'intégralité des entretiens réalisés avec certains des participants de l'étude ;2) la deuxième étape est le dépouillement des informations reçues à partir des questionnaires adressés aux étudiants et aux enseignants d'EPS. Elle a permis de faire la lumière sur les contenus des feedbacks et surtout sur leur nature. Il s'agit d'une analyse qualitative et quantitative des données recueillies.

\subsubsection{L'analyse quantitative et qualitative des données}

L'analyse quantitative des données a permis de dénombrer les différentes réponses du questionnaire, en vue de dégager la prévalence. Quant à l'analyse qualitative des données recueillies, elle a permis d'accéder au sens, à la signification que donnent les réponses des étudiants et des enseignants en rapport au questionnaire et aux questions lors des interviews d'une part avec les conseillers pédagogiques, les inspecteurs pédagogiques de l'EPS et d'autre part les enseignants chercheurs de l'INJEPS.

Notre méthode d'analyse qualitative se fonde sur un système de protocoles mettant en correspondance deux corpus. Elle résulte de la mise en tension des informations issues des questionnaires et des éléments du discours des autres participants que sont les conseillers pédagogiques, les inspecteurs pédagogiques de l'EPS et les enseignants chercheurs de l'INJEPS. Il s'agit fondamentalement d'un croisement à partir d'une analyse thématique.

\section{Les résultats}

La présentation des résultats sera focalisée essentiellement sur le contenu des feedbacks avec un accent sur la nature des savoirs qu'ils véhiculent d'une part, le déroulement de ces feedbacks et, d'autre part leur visées pédagogique et administrative. 


\subsection{Le contenu des feedbacks: vers une différenciation des variables observées}

Tableau I : Contenu des feedbacks

\begin{tabular}{|c|c|c|c|c|c|c|c|}
\hline \multicolumn{2}{|c|}{$\begin{array}{l}\text { Promotions } \\
\text { Contenu des feedbacks }\end{array}$} & $\begin{array}{c}\text { Licence } \\
1\end{array}$ & $\begin{array}{l}\text { Licence } \\
2\end{array}$ & Licence & Master1 & Master2 & Observations \\
\hline \multirow{2}{*}{$\begin{array}{l}\text { Tenue et } \\
\text { matériel du } \\
\text { stagiaire }\end{array}$} & $\begin{array}{c}\text { Tenue } \\
\text { vestimentaire }\end{array}$ & 10 & 09 & 05 & 04 & 02 & \multirow{2}{*}{$\begin{array}{c}\text { L'accent est } \\
\text { essentiellement mis sur cet } \\
\text { élément, de la Licence1 en } \\
\text { licence }\end{array}$} \\
\hline & $\begin{array}{l}\text { Matériel de } \\
\text { l'enseignant }\end{array}$ & 10 & 10 & 06 & 01 & 01 & \\
\hline \multicolumn{2}{|c|}{$\begin{array}{l}\text { Préparation et tenue des } \\
\text { documents pédagogiques } \\
\text { (contenu des documents) }\end{array}$} & 10 & 10 & 10 & 10 & 10 & $\begin{array}{l}\text { Cet élément est } \\
\text { déterminant tout au long } \\
\text { du cursus de formation }\end{array}$ \\
\hline \multirow{4}{*}{$\begin{array}{l}\text { Organisatio } \\
\text { n spatiale, } \\
\text { matérielle } \\
\text { et } \\
\text { temporelle } \\
\text { du groupe- } \\
\text { classe }\end{array}$} & $\begin{array}{l}\text { Aménagement des } \\
\text { infrastructures }\end{array}$ & 10 & 10 & 09 & 02 & 02 & $\begin{array}{l}\text { L'accent est mis sur cet } \\
\text { élément de la Licence1 en } \\
\text { Licence }\end{array}$ \\
\hline & $\begin{array}{l}\text { Gestion du } \\
\text { matériel }\end{array}$ & 10 & 08 & 05 & 06 & 08 & \multirow{3}{*}{$\begin{array}{c}\text { Cet élément est } \\
\text { déterminant tout au long } \\
\text { de la formation }\end{array}$} \\
\hline & Gestion du temps & 10 & 08 & 05 & 06 & 08 & \\
\hline & $\begin{array}{l}\text { Gestion du groupe- } \\
\text { classe }\end{array}$ & 10 & 10 & 10 & 10 & 10 & \\
\hline \multirow[t]{4}{*}{$\begin{array}{l}\text { Gestion de } \\
\text { la séance }\end{array}$} & $\begin{array}{c}\text { Respect des grands } \\
\text { principes } \\
\text { méthodologiques }\end{array}$ & 10 & 10 & 10 & 08 & 10 & $\begin{array}{l}\text { Cet élément est observé } \\
\text { régulièrement et de façon } \\
\text { méthodique durant tout le } \\
\text { cycle de formation }\end{array}$ \\
\hline & $\begin{array}{l}\text { Respect de la } \\
\text { démarche } \\
\text { d'Ens/App/Eval }\end{array}$ & 03 & 09 & 10 & 10 & 10 & $\begin{array}{l}\text { Plus observé à l'exception } \\
\text { de la Licence } 1 \text {, il est } \\
\text { observé de la Licence2 en } \\
\text { Licence } \\
\end{array}$ \\
\hline & Qualité de la voix & 10 & 10 & 05 & 02 & 02 & $\begin{array}{l}\text { Il est essentiellement } \\
\text { observé jusqu'en licence } \\
\text { et ensuite considéré } \\
\text { comme acquis }\end{array}$ \\
\hline & $\begin{array}{c}\text { Placement et } \\
\text { déplacement de } \\
\text { l'enseignant } \\
\end{array}$ & 10 & 10 & 07 & 08 & 08 & $\begin{array}{c}\text { Constant dans le suivi et } \\
\text { l'encadrement }\end{array}$ \\
\hline \multirow[t]{2}{*}{$\begin{array}{l}\text { Feedbacks } \\
\text { et conseils }\end{array}$} & $\begin{array}{l}\text { Justific } \\
\text { ation } \\
\text { des } \\
\text { choix } \\
\text { pédago } \\
\text { giques }\end{array}$ & 07 & & 08 & 09 & 10 & \multirow[t]{2}{*}{$\begin{array}{l}\text { Très important } \\
\text { dans la formation } \\
\text { de l'étudiant, c'est } \\
\text { un critère de } \\
\text { correction }\end{array}$} \\
\hline & $\begin{array}{l}\text { Conseil } \\
\text { s des } \\
\text { supervi } \\
\text { seurs }\end{array}$ & 10 & & 10 & 10 & 10 & \\
\hline
\end{tabular}

L'analyse de ce tableau montre que les contenus des feedbacks sont fonction du niveau de classe. En licence 1 un accent fondamental est mis sur les variables de contexte, de programme et de produit (le matériel, la préparation et tenue des documents pédagogiques etc..). Les variables processus ne sont pas trop intervenues dans l'évaluation. La présence de ces 
critères témoigne de la nécessité pour l'étudiant de la première année de maîtriser ces variables. Rappelons que le milieu d'intervention de l'étudiant de licencel est l'école primaire.

Par contre en licence 2, tous les critères sont pris en compte pour l'évaluation. Ils montrent que l'étudiant de la licence 2 a engrangé un peu d'expérience pouvant lui permettre de dérouler convenablement une séquence d'EPS. Il doit donc non seulement maitriser les variables de contexte mais aussi les variables de programme, de processus et de produit.

En licence, certains éléments sont moins notés que d'autres mais en proportion moyenne. Il s'agit des éléments comme : la tenue et le matériel du stagiaire ; la gestion du matériel ; la gestion du temps ; la qualité de la voix. Ils sont considérés comme des éléments déjà acquis en Licence 1 et en Licence 2. Par conséquent, les éléments essentiels de ce niveau de classe sont ceux ayant trait à la pratique enseignante. Autrement dit, les variables de programme, de processus et de produit sont les plus notées au détriment des variables de contexte

En Master 1 et en Master 2 certains de ces éléments d'évaluation sont considérés comme acquis par l'étudiant. Il s'agit des éléments comme : la tenue et matériel du stagiaire, l'aménagement des infrastructures et la qualité de la voix. On en déduit que le plus important en Master, c'est de réaliser une séance d'EPS avec aisance et en respectant tous les principes pédagogiques de l'enseignement. Les variables les plus notées sont donc les variables de programme, de processus et de produit.

Somme toute, il apparait que par promotion, certains éléments sont plus valorisés que d'autres mais toujours dans l'optique de permettre à l'enseignant d'avoir une maitrise des connaissances pédagogiques et didactiques.

Rappelons-le, la supervision pédagogique de ces étudiants est assurée par des superviseurs pédagogiques provenant de deux institutions différentes, notamment de l'institution garante de la formation initiale (l'INJEPS) et de l'IGPM, partenaire fournissant un appui technique. Quoi que les deux types de superviseurs interviennent en supervision pédagogique, il semble qu'ils utilisent des outils différents. Qu'en est-il de ces outils ? Et quels sont les critères utilisés pour l'évaluation? 
5.2. Contenus des fiches et critères d'évaluation : des éléments de disparités à pointer.

Tableau II : Étude comparative des fiches d'inspection des différentes structures de la supervision pédagogique

\begin{tabular}{lcl}
\hline $\mathrm{N}^{\circ}$ & INJEPS & IGPM \\
\hline $1-$ & Tenue et matériel du & Analyse de la prestation de l'enseignant \\
stagiaire & $\begin{array}{l}\text {-Maitrise des connaissances } \\
\text {-respect de la méthodologie } \\
\text {-progression dans l'exécution des programmes } \\
\text {-gestion de la classe : aspects relationnels } \\
\text {-autres observations }\end{array}$ \\
& Recommandations \\
\hline 3- & $\begin{array}{c}\text { Préparation et tenue des } \\
\text { documents pédagogiques } \\
\text { (contenu des documents) } \\
\text { Organisation spatiale, } \\
\text { matérielle et temporelle du } \\
\text { groupe-classe }\end{array}$ & Appréciation générale du Conseiller Pédagogique \\
$4-\quad \begin{array}{c}\text { Gestion de la séance } \\
\text { Feedbacks et conseils }\end{array}$ & Impressions de l'enseignant \\
\hline
\end{tabular}

De façon globale, le tableau 2 résume le contenu des fiches d'évaluation par structure d'encadrement. On peut en déduire que les deux institutions ont un mode d'évaluation qui diffère dans le fond plus précisément au niveau des critères ayant rapport à l'utilisation du matériel et à la gestion des infrastructures. En observant de plus près, nous notons que la fiche d'inspection de l'INJEPS est plus exhaustive car elle est destinée à l'évaluation des étudiants en formation initiale. Celle de l'IGPM est globale et destinée aux enseignants professionnels. Notons que pour l'INJEPS les variables les plus évaluées sont les variables de contexte, de programme, de processus et de produit contrairement aux inspecteurs de l'IGPM qui ne tiennent pas compte des variables de contexte. Les différentes grilles d'observation utilisées font recours à des critères qui renvoient à des variables bien précises. Quels sont ces critères d'évaluation et quelle est leur pertinence?

Tableau III: Les critères d'évaluation

\begin{tabular}{lccc}
\hline Critères & Nombre & Pourcentage\% & Total \\
\hline Assiduité aux cours & 100 & 100 & 100 \\
Tenue vestimentaire & 60 & $60 \%$ & 100 \\
Maitrise des connaissances & 97 & $97 \%$ & 100 \\
Documents pédagogiques & 100 & $100 \%$ & 100 \\
$\begin{array}{l}\text { Aménagement de l'espace et gestion } \\
\text { du matériel }\end{array}$ & 46 & $46 \%$ & 100 \\
$\begin{array}{l}\text { Gestion de la classe } \\
\text { Progression dans l'exécution des }\end{array}$ & 100 & $100 \%$ & 100 \\
programmes & 100 & $100 \%$ & 100 \\
Démarche méthodologique & 84 & $84 \%$ & 100 \\
Placement et déplacement & 86 & $86 \%$ & 100 \\
\hline
\end{tabular}




\begin{tabular}{llll}
\hline Justification des actions & 74 & $74 \%$ & 100 \\
Voix de l'enseignant & 58 & $58 \%$ & 100 \\
Autres & 43 & $43 \%$ & 100 \\
\hline
\end{tabular}

Il ressort de ce tableau que $100 \%$ des étudiants sont notés sur l'assiduité aux cours, les documents pédagogiques, la gestion de la classe et la progression dans l'exécution des programmes. $60 \%$ de ces étudiants sont notés sur la tenue pédagogique, $97 \%$ sur la maitrise des connaissances, $46 \%$ sur l'aménagement de l'espace et la gestion du matériel, $84 \%$ sur la démarche méthodologique, $86 \%$ sur le placement et déplacement, $74 \%$ sur la justification des actions, $58 \%$ sur la voix de l'enseignant et $43 \%$ sur d'autres critères. On en déduit que les variables les plus évaluées sont les variables de contexte, de programme, de processus et de produit.

\subsection{Déroulement des feedbacks}

\begin{tabular}{lccc} 
& \multicolumn{4}{c}{ Tableau IV : Déroulement des feedbacks } & \\
\hline Actions du superviseur & Nombre & Pourcentage\% & Total \\
\hline $\begin{array}{l}\text { Le superviseur fait des } \\
\text { observations }\end{array}$ & 100 & $100 \%$ & 100 \\
$\begin{array}{l}\text { Le superviseur donne des } \\
\text { conseils }\end{array}$ & 100 & $100 \%$ & 100 \\
$\begin{array}{l}\text { Le superviseur insulte } \\
\text { l'enseignant }\end{array}$ & 12 & $12 \%$ & 100 \\
$\begin{array}{l}\text { Le superviseur pose des } \\
\text { questions et laisse }\end{array}$ & 87 & $87 \%$ & 100 \\
$\begin{array}{l}\text { l'enseignant se justifier } \\
\text { Le superviseur monopolise la } \\
\text { parole } \\
\text { Autres }\end{array}$ & 08 & $08 \%$ & 100 \\
\hline
\end{tabular}

Il ressort de ce tableau que $100 \%$ des étudiants estime que le superviseur leur fait des observations et leur donne des conseils; $87 \%$ estime que le superviseur pose des questions et laisse l'enseignant se justifier. Par contre, $12 \%$ pense que le superviseur insulte l'enseignant et $08 \%$ soutient que le superviseur monopolise la parole. Nous déduisons que la plupart des superviseurs font des observations par rapport aux actions didactiques menées par le stagiaire. Ils laissent le stagiaire se justifier par rapport à ses actions et lui donnent des conseils. Ils mènent des actions conformes aux visées de supervision.

\subsection{La Gestion des feedbacks par les acteurs de la supervision : éléments de discussion}

Les modalités de déroulement et de gestion des feedbacks telles que mises en place par les acteurs des deux institutions auxquelles s'assujettissent 
les étudiants de l'INJEPS en formation initiale mettent en évidence des visées à la fois pédagogiques, administratives et structurelles.

Si l'analyse du déroulement des feedbacks met en évidence une procédure interactive entre le superviseur et le supervisé, il n'en demeure pas moins que la justification des actes`et l'ordonnance des conseils constituent les trames essentielles de cette analyse du vécu pédagogique. Il semble donc que quel qu'en soit l'acteur les procédures de déroulement des feedbacks ne diffèrent point.

Relativement aux outils utilisés, le contraste observé serait dû à la différenciation des cibles sujettes à la supervision : des étudiants en formation initiale et des enseignants en formation continue. Toutefois, dans une visée de professionnalisation continue, n'est-il pas indispensable que les deux acteurs s'accordent sur la nature des outils d'évaluation, si tant est-il que la nature des instruments utilisés influence les résultats obtenus (Brunelle et $\mathrm{al}$, 1988).

L'analyse des résultats a par ailleurs montré que le principal outil utilisé (la grille d'observation) est en lien avec les différentes variables de l'intervention (Brunelle et $a l$ ). Aussi, l'observation de ces variables par le superviseur semble être liée au niveau d'étude de l'étudiant. L'élaboration d'un standard semblerait alors nécessaire car non seulement, il fournirait des indications précises en termes d'orientation sélective de l'intervention mais aussi et surtout l'élaboration d'une grille de pondération des variables observées.

Quant aux visées pédagogiques des feedbacks, tous les acteurs s'accordent sur sa pertinence dans l'amélioration de la pratique enseignante de l'étudiant. Toutefois son impact dans la formation de l'étudiant semble très peu visible.

En effet, les rapports produits par les superviseurs au-delà du fait qu'ils précisent les différentes observations et attribuent le score (notes), ne s'inscrivent aucunement dans une visée de formation. Ils ne sont pas exploités pour des remédiations et dans la définition des syllabi. Or ils sont révélateurs des difficultés des étudiants aux plans pédagogique et didactique.

$\mathrm{Au}$ plan administratif, la note obtenue des feedbacks participe au passage en année supérieure des étudiants car cette note entre en ligne de compte pour le calcul de la moyenne en pédagogie qui constitue une unité d'enseignement très importante dans la professionnalisation du stagiaire.

\section{Discussion}

Les étudiants stagiaires sont astreints au cours de leurs stages pédagogiques, à deux catégories de superviseurs pédagogiques utilisant chacune des grilles d'évaluation différentes suivant leur niveau d'étude. Les résultats obtenus, s'ils témoignent des conditions de supervision pédagogique des étudiants en formation initiale et de déroulement des feedbacks mettent 
aussi au jour les dysfonctionnements dans l'organisation de ces stages et surtout dans les issues accordées aux résultats des feedbacks. Les différents feedbacks, s'ils ont permis aux stagiaires investis l'ajustement de leur posture pédagogique voire didactique, semblent ne pas aider à l'atteinte de l'une des ambitions de changement de la professionnalisation qu'est la formation (Maubant et $a l, 2013$ ); le volet formation étant un maillon très fort de du processus de construction de la professionnalisation.

Relativement à l'organisation, il nous semble indispensable selon le niveau du stagiaire de mettre en place un dispositif d'accompagnement pédagogique dès les premières années pédagogiques de l'étudiant dans la perspective de limiter la peur que suscite parfois l'inspection pédagogique (Brunel et al ,1988). La professionnalisation étant entre autres liée à l'analyse de l'exercice des exigences du métier (Altet, 2002), une étude exploratoire dans ce sens permettrait une probable amélioration de ce dispositif et faine ainsi un clin d'œil à la didactique professionnelle.

La situation de psychose que provoque l'inspection pédagogique au niveau des étudiants stagiaires, montre que les résultats obtenus s'inscrivent aux antipodes d'une dédramatisation de l'inspection pédagogique (Opéra, 2017). Ainsi pour limiter la peur qui anime les étudiants avant et pendant la supervision pédagogique, l'adoption d'une démarche coopérative et l'instauration d'un climat propice des échanges constructifs pour le développement et l'auto-développement professionnel des stagiaires lors des supervisions, semblent être indispensable pour les visées d'une inspection pédagogique constructive. Passer d'une posture d'inspecteur évaluateur à une posture de superviseur conseil et d'assistance favoriserait la construction d'une potentielle professionnalité.

L'autre aspect que soulèvent aussi ces résultats obtenus relativement à l'organisation et à l'utilisation des feedbacks issus des inspections est celui de la conception d'un environnement de formation énactée (Pozat et $a l$ ). Une approche conceptuelle de l'environnement de la formation devrait concilier l'appropriation de nouveaux objets issus de l'analyse des feedbacks et aussi de la structuration des contenus de formation avec l'exercice du métier (le futur enseignant d'EPS).

Par ailleurs, si la conduite des feedbacks pédagogiques par les deux types de superviseurs des deux institutions rencontre l'assentiment des étudiants stagiaires (Cf. résultats), il faut toutefois pointer l'inexistence d'un creuset de dialogue pédagogique entre ces deux institutions gage d'un encadrement efficace et diversifié. Même si ces dernières ne visent pas les mêmes objectifs (l'une en charge de la formation initiale : INJEPS ; l'autre en charge de la formation continue : DIP), leurs actions pourraient s'inscrire dans une synergie totale. Il est vrai que les autorités de l'INJEPS semblent s'inscrire dans cette dynamique lorsqu'elles ont pensé associer les inspecteurs de la DIP, 
mais la collaboration semble souffrir d'une redynamisation et d'un peu de résistance.

\section{Conclusion}

L'objectif de cette étude est d'analyser, d'une part, au plan pédagogique le système d'évaluation de la pratique enseignante des stagiaires afin de comprendre sa pertinence et d'autre part, au plan administratif de comprendre comment ces feedbacks sont utilisés en vue d'améliorer la qualité de l'enseignement. Pour l'atteindre, nous avons formulé une problématique fondée sur un cadre théorique composite reposant sur deux modèles d'analyse ; celui de Dunkin et Biddle (1974) adapté par Brunelle et al. (1988) et sur le modèle anthropologique du didactique de Chevallard (1992). Cette problématique nous a permis de formuler quatre questions de recherche auxquelles nous avons répondu à partir d'une démarche méthodologique fondée sur un questionnaire et un entretien avec différents acteurs à savoir : les superviseurs de l'IGPM et de l'INJEPS, les étudiants et les membres de l'administration de l'INJEPS.

Des résultats, il ressort généralement que:

- la plupart des visites de classe effectuées aux étudiants sont faites par les enseignants-chercheurs de l'INJEPS et de façon périodique. Il s'agit à l'INJEPS de conseillers pédagogiques hautement qualifiés non seulement en matière d'enseignement et de supervision, mais aussi en matière de recherche. Ils utilisent dans cette optique une grille d'évaluation composée de critères observables et objectifs reflétant le niveau de compétence de l'étudiant.

- A la fin de la prestation de l'étudiant, une séance d'entretien avec le superviseur est initiée. Il s'agit du feedback lors duquel la prestation de l'étudiant est critiquée avec l'apport de quelques conseils qu'il prend la peine de transcrire dans son cahier de bilan. Ces conseils seront utilisés pour améliorer sa pratique pédagogique.

- Le même processus est observé avec les inspecteurs de l'IGPM qui à la différence des enseignants de l'INJEPS sont qualifiés pour inspecter les enseignants en fonction. Les rapports issus des feedbacks de cette inspection permettent aux enseignants de monter théoriquement en grade. Mais lorsqu'ils sont sollicités pour inspecter les étudiants, leur méthode de supervision rejoint celle des enseignants de l'INJEPS dont le but est de donner une note chiffrée à la prestation de ces étudiants.

- Les savoirs transmis par les superviseurs et acquis par les stagiaires lors des entretiens post-enseignement, sont de nature «pratico-technique et technologico-théorique » en réfléchir à Chevallard (1999). Ils varient en fonction des superviseurs et de la prestation des supervisés; d'où la variance de la nature des remarques et observations des conseillers pédagogiques, entraînant des notes subjectives dont l'objectivation fera l'objet 
d'investigations à venir. Lors des feedback, les entretiens se déroulés de façon non directive et les objets de savoir de ces entretiens sont centrés sur les variables de contexte, de programme, de processus et de produit.

\section{Limites et perspectives}

Il faut toutefois reconnaître qu'au plan pédagogique très peu de dispositions sont prises pour pallier cette situation où les attendus des feedbacks ne sont pas exploités. A défaut de constituer des objets de formation ou de renforcement du substrat pédagogique des étudiants stagiaires, une synthèse d'analyse des rapports issus des feedbacks des inspections pédagogiques devrait être disponible dans les archives pédagogiques de la Direction en charge des stages pédagogiques. Malheureusement ce vide pédagogique est constaté. Dans la perspective d'un prolongement de cette étude, il faut prendre en compte, non seulement cet aspect mais aller vers une ingénierie didactique fondée sur la conduite des feedbacks pédagogiques.

\section{References:}

1. Albanel, X. (2009). Le travail d'évaluation : L'inspection dans l'enseignement secondaire. Toulouse : Octares Editions.

2. Alexis P. (2012). Perceptions Et Pratiques De La Supervision Pédagogique Au Niveau Primaire (Premier Et Deuxième Cycles Fondamental) En Haïti. Mémoire pour l'obtention du grade de Maître ès arts (M.A.). Institut de formation des Maîtres de Port au Prince.

3. Altet, M. (2002). Une démarche de Recherche sur la pratique enseignante : l'analyse plurielle, Revue Française de pédagogie, ${ }^{\circ}$ $138,85-93$.

4. Alliance des professeurs de Montréal (2005). Les fiches syndicales, la supervision pédagogique. [En ligne]. Consulté le 6 Mai 2017. Disponible http://www.comprof.ca/communications/supervision\%20pedagogiqu e\%20formelle.pd f

5. Attiklémé K., Kpazaï G. (2010). Approche didactique de l'entretien postenseignement entre le superviseur pédagogique et le stagiaire : cas de la formation pratique en enseignement de l'Education Physique et Sportive au secondaire a l'INJEPS de Porto-Novo du Benin. Analele Steintifice ale Universitatii Alexandru Ioan Cuza-Sect. Stuintele Educatiel, vol. XIV, pp. 242-260

6. Attiklémé, K. (1994). Supervision de l'enseignement de l'EPS au Bénin : Situation actuelle et perspectives. Mémoire pour l'obtention du Certificat d'Aptitude à l'Inspection de la Jeunesse et des Sports, Mémoire INJS Abidjan, Côte d'Ivoire, p. 99. 
7. Awal. I. (2004). La supervision de l'enseignement de l'EPS dans le département de l'Ouémé : quel système d'évaluation pour améliorer un tel enseignement ? Mémoire de maîtrise, INJEPS. Porto-Novo, Bénin, p.30

8. Bouchamma, Y. (2004). Supervision de l'enseignement et réformes scolaires. Conférence présentée au Biennale de l'éducation, Lyon, France.

9. 9-Baillat, G., Niclot, D., \& Ulman, D ;52010 ; La formation des enseignants en Europe: Approche comparative. Pédagogie en développement. De Boeck supérieur.

10. Bouchamma Y., Giguère M., April D. (2016). La supervision pédagogique : Guide pratique à l'intention des directions et des directions adjointes des établissements scolaires, Université Laval (Canada), p.12

11. Bouchamma, Y. (2004) Supervision de l'enseignement et réformes. [En ligne]. Consulté le 6 Mai 2017. Disponible : http://www.inrp.fr/biennale/7biennale/Contrib/longue/7300.pdf

12. Boutet, N. \& Rousseau, M. (2002). Les enjeux de la supervision pédagogique des stages. Ste Foy : Presses de l'Université du Québec.

13. Brunelle. J., Drouin, D., Godbout, P., Tousignant, M. (1988). La supervision de l'intervention en activité physique. Montréal : Gaëtan Morin Éditeur.

14. Carlier G. (2002). Superviser des stagiaires en Education physique : balises pour une fonction en voie de professionnalisation. Avante Université catholique de Louvain(Belgique). Vol.8, No1, pp.96-111

15. Chevallard. Y. (1991). La transposition didactique du savoir savant au savoir enseigné. Grenoble, la pensée sauvage, (2ème édition augmentée).

16. Chevallard. Y. (1999). L'analyse des pratiques enseignantes en théorie anthropologique du didactique. Recherche en didactique des Mathématiques, $n^{\circ} 2,221-266$.

17. Commission scolaire de Montréal (2005). Supervision pédagogique. [En ligne]. Consulté le 9 Mai 2012. Disponible : http://www.comprof.ca/communications/supervision\%20pedagogiqu e\%20formelle.pd f

18. Circulaire $n^{\circ}$ 2009-064 du 19-5-2009. Missions des corps d'inspection : inspecteurs d'académie-inspecteurs pédagogiques régionaux et inspecteurs de l'Éducation nationale affectés dans les académies, (BO n²2 du 28 mai 2009)

19. Dunkin, M. et Biddle, B.J. (1974). The study of teaching. New York, Holt Rinechart Whinston. 
20. Glatthorn, A. (1984). Differentiated Supervision. Virginia, USA: Association for Supervision and Curriculum Development.

21. Issaka A. (2005). La Supervision Pédagogique en EPS au Niger, Situation actuelle et perspectives : cas des établissements secondaires publics de la région de Niamey. Mémoire pour l'obtention du Certificat d'Aptitude aux fonctions d'inspecteur de l'Education Populaire de la Jeunesse et des Sports C.A.I.E.P.J.S. pp.19-23 INSEPS ; Dakar.

22. Legendre R. (2005). Dictionnaire actuel de l'éducation, 3e édition. Montréal : Guérin éditeur.

23. Poizat, G., Salin, D. \& Durand, M. (2013). Approche énactive humaine, simplexité et conception de formations professionnelles. Education Science et Société ; Vol4, n 1 pp.108-110.

24. Maubant, P., Roger, L. ， \& Lejeune, M. (2013). Déprofessionnalisation. Recherche et formation. 72(2041

25. Nolan, J. F., \& Hoover, L. A. (2010). Teacher supervision \& evaluation: theory into practice (3e éd.). Hoboken, NJ: Wiley.

26. Oliva, P. F., \& Pawlas, G. (2004). Supervision for today's schools (7 e éd.). New York : Wiley. Etats Unis

27. Opéra (2017). L'accompagnement professionnel des enseignants du fondamental (élémentaire et collège) en Afrique subsaharienne ; AUF.

28. Parlebas, P. (1981). Contribution à un lexique commenté en science de l'action motrice. Paris : Publications I.N.S.E.P

29. Sergiovanni, T. J., \& Starratt, R. J. (2007). Supervision: a redefinition (8e éd.). Boston, MA : McGraw-Hill.

30. Sheedy, A. (1980-1981). Les principes de l'Education Physique Revue des Sciences de l'éducation et de formation voll $\mathrm{n}^{\circ} 15 \mathrm{p}$ 1-6.

31. Snow-Gerono, J. L. (2008). Locating Supervision-A Reflective Framework for Negotiating Tensions within Conceptual and Procedural Foci for Teacher Development. Teaching and Teacher Education, 24(6), 1502-1515.

32. Sogbossi, F. (1994). Evaluation des feed-back émis par les enseignants d'EPS en situation d'enseignement apprentissage dans les établissements secondaires de Cotonou et Porto-Novo. Mémoire de CAPEPS, INEEPS, Porto-Novo, Bénin, p 58.

33. Sullivan, S., \& Glanz, J. (2004). Supervision that Improves Teaching: Strategies and Techniques (2e éd.). Thousand Oaks, CA : Corwin Press.

34. Villeuneuve, L. (1994). L'encadrement du stage supervisé. Éditions Saint-Martin, Montréal Québec 